J. Appl. Numer. Optim. 1 (2019), No. 1, pp. 39-51

Available online at http://jano.biemdas.com

https://doi.org/10.23952/jano.1.2019.1.04

\title{
CONVERGENCE OF SOLUTIONS TO LEXICOGRAPHIC EQUILIBRIUM PROBLEMS
}

\author{
L.Q. ANH ${ }^{1, *}$, T. BANTAOJAI ${ }^{2}$, N.P. DUC ${ }^{3,4}$, T.Q. DUY ${ }^{5}$, R. WANGKEEREE ${ }^{6}$ \\ ${ }^{1}$ Department of Mathematics, Teacher College, Can Tho University, Can Tho, Vietnam \\ ${ }^{2}$ Valaya Alongkorn Rajabhat University under the Royal Patronage, Pathumtani, Thailand \\ ${ }^{3}$ Faculty of Mathematics and Computer Science, University of Science, \\ Vietnam National University, Ho Chi Minh City, Vietnam \\ ${ }^{4}$ Department of Mathematics, Kien Giang Technical and Economic College, Kien Giang, Vietnam \\ ${ }^{5}$ Department of Mathematics, FPT University, Can Tho, Vietnam \\ ${ }^{6}$ Department of Mathematics, Faculty of Science, Naresuan University, Phitsanulok, Thailand
}

\begin{abstract}
This article deals with lexicographic equilibrium problems on Banach spaces. We first study the existence of solutions for such problems. Then, we investigate the Painlevé-Kuratowski convergence of the solution sets for a family of perturbed problems in a such way that they are perturbed by sequences constrained sets and objective functions converging. Several illustrative examples are given which clarify the essentialness of imposed assumptions. As an application, we discuss various results on the Painlevé-Kuratowski convergence for lexicographic variational inequalities.

Keywords. Lexicographic order; Equilibrium problem; Variational inequality; Painlevé-Kuratowski convergence; Continuous convergence.
\end{abstract}

2010 Mathematics Subject Classification. 49M37, 90C30, 47J20.

\section{INTRODUCTION}

The vector equilibrium problem contains many problems as special cases, including vector variational inequalities, vector optimization problems, vector complementarity problems, vector Nash equilibrium problems, etc. Because of the general form of this problem, in fact it was investigated earlier under other terminologies (see, e.g., $[10,14,26])$. Most works on vector variational inequalities and vector equilibrium problems are based on orders induced by convex closed cones, i.e., they used various extensions of the Pareto order. However, it is known from the theory of vector optimization that the set of Paretooptimal points is usually too large, so that one needs certain additional rules to reduce it. One of the possible approaches is to utilize the lexicographic order, which was investigated in connection with its applications in optimization and decision making theory. Konnov [18] studied vector equilibrium problems using the lexicographic order and showed that several classes of inverse lexicographic optimization problems can be reduced to lexicographic vector equilibrium problems. In [8], Bianchi, Konnov and Pini established several existence results for lexicographic variational inequalities by using equivalence

\footnotetext{
${ }^{*}$ Corresponding author.

E-mail addresses: quocanh@ctu.edu.vn (L.Q. Anh), thanatpornmaths@gmail.com (T. Bantaojai),ngphucduc.kg@gmail. com (N.P. Duc), duytq4@fe.edu.vn (T.Q. Duy), rabianw@nu.ac.th (R. Wangkeeree).

Received February 17, 2019; Accepted March 21, 2019.
}

(C)2019 Journal of Applied and Numerical Optimization 
properties between various kinds of lexicographic variational inequalities and sequential ones. Bianchi, Konnov and Pini [9] discussed lexicographic equilibrium problems on a topological Hausdorff vector space and their relationships with some other vector equilibrium problems. They established existence results for the tangled lexicographic problem via the study of a related sequential problem. Anh, Duy and Khanh [3] established the sufficient conditions for the upper semicontinuity, closedness, and continuity of solution maps for a parametric lexicographic equilibrium problem. The topic of well-posedness for problems involving lexicographic cones has received attention by many researchers recently; see, e.g., $[2,4]$. Very recently, Anh and Duy [5] proposed a penalty function method for solving the lexicographic equilibrium problem. They use regularized gap function to solve such penalized problem and establish an error bound result for the penalized problem.

Set convergence, which is based on the limits of sets, was introduced by Painlevé (between 1902 and 1905) and popularized by Kuratowski in his book [19] in 1968, and thus often called the PainlevéKuratowski convergence. To date, it has been viewed as a major tool for dealing with approximations of variational problems in optimization, systems of equations and related objects. This concept plays an important role in the stability theory of vector optimization and its applications. It gives us the relationships between cluster points of approximating solutions set and exact solutions to the original problem, and hence it is the bridge among stability, well-posedness and solution method topics. Since problem$\mathrm{s}$ in terms of asymptotically solving sequences are different from such parametric problems with the parameters perturbed in spaces of parameters, investigation of Painlevé-Kuratowski convergence of approximate solution sets is significant. Therefore, this topic has attracted attention of many researchers all over the world. There are many publications concentrating on this topic for problems related to optimization, such as vector optimization problems [11, 12, 20, 25, 27, 29], vector variational inequalities [23, 24], and recently, this topic has been also discussed on vector equilibrium problems; see, e.g., $[13,21,22]$. It is worth noting that since the lexicographic cone is neither closed nor open, the results obtained in [13, 21, 22] cannot be applied to lexicographic equilibrium problems as the ordered cones in these papers are required to be closed. Moreover, to our knowledge, there is no work devoted to the Painlevé-Kuratowski convergence for lexicographic equilibrium problems.

Motivated and inspired by research works mentioned above, the aim of this article is to study the existence of solutions to lexicographic equilibrium problem and the Painlevé-Kuratowski convergence of solutions for a family of perturbed problems. Using the continuous convergence of bifunction sequences and the Painlevé-Kuratowski convergence of set sequences, we obtain the Painlevé-Kuratowski convergence of the approximate solution sets for perturbed lexicographic equilibrium problems to the solution set for the original problem. In addition, we also consider the special case of lexicographic variational inequalities as an application.

This paper is organized as follows. In Section 2, some preliminary notions and results are recalled. Section 3 is devoted to the study of existence of solutions for lexicographic equilibrium problems. The Painlevé-Kuratowski convergence of solution sets for perturbed lexicographic equilibrium problems to corresponding solution set of the original problem is established in Section 4. In the last section, Section 5, several results on the Painlevé-Kuratowski convergence for lexicographic variational inequalities are derived from the obtained results as an application. 


\section{PRELIMINARIES}

Throughout this article, unless specified otherwise, let $X$ be a Banach space with its dual space $X^{*}$. The norm and the dual pair between $X$ and $X^{*}$ are denoted respectively by $\|\cdot\|$ and $\langle\cdot, \cdot\rangle$. We recall that given a sequence of subsets $\left\{K_{n}\right\}$ in $X$, the Painlevé-Kuratowski upper and lower limits are defined, respectively, as

$$
\begin{gathered}
\limsup _{n \rightarrow \infty} K_{n}:=\left\{x \in X \mid x=\lim _{k \rightarrow \infty} x_{n_{k}}, x_{n_{k}} \in K_{n_{k}}, n_{k} \text { a selection of the integers }\right\} ; \\
\liminf _{n \rightarrow \infty} K_{n}:=\left\{x \in X \mid x=\lim _{n \rightarrow \infty} x_{n}, x_{n} \in K_{n} \text { for } n \text { sufficiently large }\right\} .
\end{gathered}
$$

In the literature, the condition $\limsup _{n \rightarrow \infty} K_{n} \subset K \subset \liminf _{n \rightarrow \infty} K_{n}$ is referred to as the convergence of $K_{n}$ to $K$ in the sense of Painlevé-Kuratowski, denoted by $K_{n} \stackrel{\text { P.K. }}{\longrightarrow} K$. The relations $K \subset \liminf _{n} K_{n}$ and $\limsup _{n} K_{n} \subset K$ are referred to as the lower part of the convergence and upper part of the convergence, respectively.

Lemma 2.1. Let $K$ be a compact subset of $X$, and $K_{n}(n=1,2, \ldots)$ be nonempty subsets of $X$ such that $\limsup _{n \rightarrow \infty} K_{n} \subset K$. Then, for every sequence $\left\{x_{n}\right\}$ with $x_{n} \in K_{n}$ there exists a subsequence $\left\{x_{n_{k}}\right\}$ converging to some point $x$ in $K$.

Proof. Suppose to the contrary that for every $x \in K$ there exist a neighborhood $V_{x}$ of $x$ and $N \in \mathbb{N}$ such that $x_{n} \notin V_{x}$ for all $n \geq N$. Because the family $\left\{V_{x}: x \in K\right\}$ is an open cover of the compact set $K$, there is a finite subcover $V=\bigcup_{i=1}^{m} V_{x_{i}}$ containing $K$. Consequently, there exists an $n_{0} \in \mathbb{N}$ such that $x_{n} \notin V$ for all $n \geq n_{0}$, which contradicts $\limsup _{n \rightarrow \infty} K_{n} \subset K$.

We also recall the notion of continuous convergence for a sequence of extended real-valued functions.

Definition 2.1. [28] A sequence of functions $\left\{f_{n}\right\}$ where $f_{n}: X \rightarrow \mathbb{R} \cup\{ \pm \infty\}$, is said to converge to a function $f: X \rightarrow \mathbb{R} \cup\{ \pm \infty\}$ in the sense of continuous convergence if and only if $f_{n}\left(x_{n}\right) \rightarrow f(x)$ for every sequence $\left\{x_{n}\right\}$ converging to $x$.

This convergence is denoted by $f_{n} \stackrel{\mathrm{c}}{\rightarrow} f$.

Definition 2.2. [15, Definition 2.5.1] Let $X$ and $Y$ be Banach spaces and $\bar{x} \in X$. A set-valued mapping $F: X \rightrightarrows Y$ is said to be

(a) upper semicontinuous (usc) at $\bar{x}$ if for any open subset $U$ of $Y$ with $F(\bar{x}) \subset U$, there is a neighborhood $N$ of $\bar{x}$ such that $F(N) \subset U$.

(b) lower semicontinuous (lsc) at $\bar{x}$ if for any open subset $U$ of $Y$ with $F(\bar{x}) \cap U \neq \emptyset$, there is a neighborhood $N$ of $\bar{x}$ such that $F(x) \cap U \neq \emptyset$ for all $x \in N$.

(c) continuous at $\bar{x}$ if it is both usc and lsc at $\bar{x}$.

We say that a mapping has a certain property on a set $A$ if so does it at every point of $A$.

Definition 2.3. [1,17] Let $K$ be a nonempty convex subset of $X$. A function $f: X \rightarrow \mathbb{R}$ is said to be

(a) upper semicontinuous at $x \in X$ if for every sequence $\left\{x_{n}\right\}$ in $X$ converging to $\mathrm{x}$, one has

$$
f(x) \geq \limsup _{n \rightarrow \infty} f\left(x_{n}\right) .
$$

(b) lower semicontinuous at $x \in X$ if $-f$ is upper semicontinuous at $x$. 
(c) quasiconvex on $K$ if for each pair of points $x, y \in K$ and for all $t \in[0,1]$, one has

$$
f(t x+(1-t) y) \leq \max \{f(x), f(y)\} .
$$

(d) strictly quasiconvex on $K$ if for each pair of points $x, y \in K, x \neq y$ and $t \in] 0,1[$, one has

$$
f(t x+(1-t) y)<\max \{f(x), f(y)\} .
$$

(e) semistrictly quasiconvex on $K$ if for each pair of points $x, y \in K, x \neq y$ such that $f(x) \neq f(y)$ and $t \in] 0,1[$, one has

$$
f(t x+(1-t) y)<\max \{f(x), f(y)\} .
$$

(f) explicitly quasiconvex on $K$ if it is quasiconvex and semistrictly quasiconvex on $K$.

(g) quasiconcave (respectively, strictly quasiconcave, semistrictly quasiconcave, explicitly quasiconcave) on $K$ if $-f$ is quasiconvex (respectively, strictly quasiconvex, semistrictly quasiconvex, explicitly quasiconvex) on $K$.

It is worth noting that there is no relationship between the class of quasiconvex functions and that of semistrictly quasiconvex ones. However, a function $f$ is explicitly quasiconvex whenever it is lower semicontinuous and semistrictly quasiconvex.

Definition 2.4. [9] Let $K$ be a nonempty subset of $X$ and $\varphi: K \times K \rightarrow \mathbb{R}$ be a bifunction.

(a) $\varphi$ is said to be pseudomonotone on $K$ if for each pair of points $x, y \in K$, the following implication holds

$$
[\varphi(x, y) \geq 0] \Longrightarrow[\varphi(y, x) \leq 0] .
$$

(b) $\varphi$ is said to be properly quasimonotone on $K$ if for every finite set $A \subset K$ and for every $x$ in the convex hull of $A$, it holds that $\max _{y \in A} \varphi(x, y) \geq 0$.

(c) $\varphi$ is said to be pseudo-symmetric on $K$ if for each pair of points $x, y \in K$, the following implication holds:

$$
[\varphi(x, y)=0] \Longrightarrow[\varphi(y, x)=0] .
$$

(d) $\varphi$ is said to be pseudomonotone ${ }_{*}$ on $K$ if it is pseudomonotone on $K$ and for each pair of points $x, y \in K$, if $\varphi(x, y)=\varphi(y, x)=0$ then for every $z \in K$, there exists a positive real number $k$ depended on $x, y, z$ such that

$$
\varphi(x, z)=k \varphi(y, z) .
$$

(e) $\varphi$ possesses the triangle inequality if

$$
\varphi(x, y) \leq \varphi(x, z)+\varphi(z, y), \quad \forall x, y, z \in K .
$$

(f) $\varphi$ is said to be a weak pseudo-distance on $K$ if for every triple $x, y, z \in K$, there exist positive real numbers $k, l$ depended on $x, y, z$ such that the following implication is satisfied

$$
[\varphi(x, y) \geq 0] \Longrightarrow[k \varphi(x, z)+l \varphi(z, y) \geq 0]
$$

It is clear that the class of weak pseudo-distance functions strictly contains that of triangle inequality functions. Now we propose a modified version of the weak pseudo-distance notion. 
Definition 2.5. Let $K$ be a nonempty subset of $X$. A bifunction $\varphi: K \times K \rightarrow \mathbb{R}$ is said to be a transfer weak pseudo-distance if $\varphi(x, y)=0$ for each pair of points $x, y \in K$ then the following implication holds

$$
[\varphi(x, z) \geq 0, \forall z \in K] \Longrightarrow[\varphi(y, z) \geq 0, \forall z \in K] .
$$

We discuss some sufficient conditions for the transfer weak pseudo-distance property.

Proposition 2.1. Suppose that $\varphi: K \times K \rightarrow \mathbb{R}$ satisfies one of the following conditions:

(i) $\varphi$ is pseudo-symmetric and pseudomonotone ${ }_{*}$.

(ii) $\varphi$ is a weak pseudo-distance.

Then, $\varphi$ is a transfer weak pseudo-distance.

Proof. The transfer weak pseudo-distance of $\varphi$ follows immediately from (i). Suppose that $\varphi$ satisfies condition (ii). Let $x, y \in K$ be such that $\varphi(x, y)=0$. Then, for each $z \in K, \varphi(x, z) \geq 0$, there exist $k, l>0$ satisfying

$$
k \varphi(x, y)+l \varphi(y, z) \geq 0 .
$$

Consequently, $\varphi(y, z) \geq 0$, which implies that $\varphi$ is a transfer weak pseudo-distance. The proof is complete.

We now recall the concept of lexicographic cone in finite dimensional space and models of equilibrium problems with the order induced by such cone. The lexicographic cone of $\mathbb{R}^{n}$, denoted $C_{\text {lex }}$, is the collection of zero and all vectors in $\mathbb{R}^{n}$ whose the first nonzero coordinate is a positive number, i.e.,

$$
C_{\text {lex }}:=\{0\} \cup\left\{x \in \mathbb{R}^{n} \mid x_{1}=\cdots=x_{j}=0, x_{j+1}>0, \text { for some } j, 0 \leq j<n\right\} .
$$

This cone is convex and pointed, and induces the total order as follow:

$$
x \geq_{L} y \Leftrightarrow x-y \in C_{\mathrm{lex}} .
$$

We also observe that it is neither closed nor open. Indeed, when comparing with the cone $C_{1}:=\{x \in$ $\left.\mathbb{R}^{n} \mid x_{1} \geq 0\right\}$, we see that int $C_{1} \subsetneq C_{\text {lex }} \subsetneq C_{1}$, while

$$
\operatorname{int} C_{\text {lex }}=\operatorname{int} C_{1} \text { and } \mathrm{cl} C_{\text {lex }}=C_{1} \text {. }
$$

Let $f:=\left(f^{1}, f^{2}\right): X \times X \rightarrow \mathbb{R}^{2}$ be a vector-valued bifunction satisfying $f(x, x)=0$ for all $x \in X$, and $K$ be a nonempty closed subset of $X$. Let us now consider the following lexicographic vector equilibrium problem.

(LEP) Find $\bar{x} \in K$ such that

$$
f(\bar{x}, y) \geq_{L} 0, \quad y \in K .
$$

The set of the solutions to the problem (LEP) is denoted by $S_{\text {LEP. }}$. We consider the lexicographic equilibrium problem under perturbations by a sequence of problems which are expressed in terms of perturbing the constraint set and the objective function. Then, (LEP) is embedded into the following family.

$(\mathrm{LEP})_{n}$ Find $x_{n} \in K_{n}$ such that

$$
f_{n}\left(x_{n}, y\right) \geq_{L} 0, \quad \forall y \in K_{n},
$$

where $f_{n}: X \times X \rightarrow \mathbb{R}^{2}$ and $K_{n}$ are nonempty subsets of $X$.

By $S_{\text {LEP }}^{n}$ we denote the solution sets of the such problems. It is worth noticing that the problem (LEP) can be stated in the following equivalent way: 
(LEP) Find $\bar{x} \in K$ such that

$$
\left\{\begin{array}{l}
f^{1}(\bar{x}, y) \geq 0, \quad \forall y \in K, \\
f^{2}(\bar{x}, z) \geq 0, \quad \forall z \in Z(\bar{x}),
\end{array}\right.
$$

where $Z: X \rightrightarrows K, Z(x):=\left\{y \in K \mid f^{1}(x, y)=0\right\}$. The problem (LEP) can clearly be rewritten as: Find $\bar{x} \in S_{\mathrm{EP}_{1}}$ such that

$$
f^{2}(\bar{x}, z) \geq 0, \quad \forall z \in Z(\bar{x})
$$

where $S_{\mathrm{EP}_{1}}:=\left\{\bar{x} \in K \mid f^{1}(\bar{x}, y) \geq 0, \forall y \in K\right\}$.

The family of perturbed lexicographic vector equilibrium problems (LEP) $)_{n}, n \in \mathbb{N}$, is restated similarly by

$(\mathrm{LEP})_{n}$ Find $x_{n} \in K_{n}$ such that

$$
\begin{cases}f_{n}^{1}\left(x_{n}, y\right) \geq 0, & \forall y \in K_{n} \\ f_{n}^{2}\left(x_{n}, z\right) \geq 0, & \forall z \in Z_{n}\left(x_{n}\right),\end{cases}
$$

where $Z_{n}: X \rightrightarrows K_{n}, Z_{n}\left(x_{n}\right):=\left\{y_{n} \in K_{n} \mid f_{n}^{1}\left(x_{n}, y_{n}\right)=0\right\}$.

\section{EXISTENCE CONDITIONS}

In this section, we establish the existence of solutions to the lexicographic equilibrium problem. Unless otherwise specified, we assume throughout this article that $K$ is a nonempty-convex-compact subset of $X$. The compactness assumption used here is only for the sake of simplifying the presentation. In fact, this assumption can be replaced by the various coercivity conditions, for more details we refer the reader to $[1,7,10]$.

We first recall some well-known results about the Kakutani-Fan-Glicksberg fixed point theorem and the Ky Fan's minimax inequality theorem.

Proposition 3.1. [16] Let A be a nonempty compact convex subset of a locally convex Hausdorff space $X$. Let $\Phi: A \rightrightarrows A$ be an upper semicontinuous mapping with nonempty closed and convex values. Then, $\Phi$ has a fixed point in $A$.

Proposition 3.2. [14] Let $\theta: K \times K \rightarrow \mathbb{R}$ be an equilibrium bifunction, i.e., $\theta(x, x)=0$ for every $x \in K$. If $\theta(\cdot, y)$ is upper semicontinuous for each $y \in K$, and $\theta(x, \cdot)$ is quasiconvex for each $x \in K$, then there exists a point $\bar{x} \in K$ such that

$$
\theta(\bar{x}, y) \geq 0, \quad \forall y \in K
$$

It is obvious that under conditions assumed in Proposition 3.2, the solutions set of the scalar equilibrium $\operatorname{EP}(K, \theta)$ is compact. To obtain some additional properties of the solutions set to equilibrium problems, we would like to recall the following results; see, for instance, $[1,6]$.

Proposition 3.3. Under assumptions of Proposition 3.2 and suppose further that:

(i) $\theta$ is pseudomonotone;

(ii) $\theta(x, \cdot)$ is explicitly quasiconvex for each $x \in K$.

Then, the solutions set of $\operatorname{EP}(K, \theta)$ is nonempty, compact and convex.

Proposition 3.4. Suppose that

(i) $\theta$ is properly quasimonotone; 
(ii) $\theta(\cdot, y)$ is upper semicontinuous for each $y \in K$;

(iii) $\theta(\cdot, y)$ is quasiconcave for each $y \in K$.

Then, the solutions set of $\mathrm{EP}(K, \theta)$ is nonempty, compact and convex.

Inspired by the Kakutani-Fan-Glicksberg fixed point theorem, we propose the following existence result for (LEP).

Theorem 3.1. Assume that the following assumptions hold:

(i) $f^{1}(\cdot, y)$ and $f^{2}$ are upper semicontinuous for each $y \in K$;

(ii) $f^{1}(x, \cdot)$ and $f^{2}(x, \cdot)$ are explicitly quasiconvex for each $x \in K$;

(iii) $f^{1}$ and $f^{2}$ are pseudomonotone;

(iv) $Z$ is lsc on $S_{\mathrm{EP}_{1}}$.

Then, the set $S_{\mathrm{LEP}}$ is nonempty and compact.

Proof. In light of Proposition 3.1, we see that $S_{\mathrm{EP}_{1}}$ is nonempty, compact and convex. For each $x \in S_{\mathrm{EP}_{1}}$, it is easy to see that $Z(x)$ is convex. Let's consider the set-valued mapping $\Phi: S_{\mathrm{EP}_{1}} \rightrightarrows S_{\mathrm{EP}_{1}}$ defined as

$$
\Phi(x):=\left\{u \in S_{\mathrm{EP}_{1}} \mid f^{2}(u, y) \geq 0, \forall y \in Z(x)\right\} .
$$

We first check the convexity of $\Phi(x)$. Let $u_{1}, u_{2} \in \Phi(x)$. Then, for all $y \in Z(x)$, we have

$$
f^{2}\left(u_{1}, y\right) \geq 0 \quad \text { and } \quad f^{2}\left(u_{2}, y\right) \geq 0 .
$$

Since $f^{2}$ is pseudomonotone,

$$
f^{2}\left(y, u_{1}\right) \leq 0 \quad \text { and } \quad f^{2}\left(y, u_{2}\right) \leq 0 .
$$

This fact together with the quasiconvexity of $f^{2}(y, \cdot)$ gives

$$
f^{2}\left(y, t u_{1}+(1-t) u_{2}\right) \leq \max \left\{f^{2}\left(y, u_{1}\right), f^{2}\left(y, u_{2}\right)\right\} \leq 0 .
$$

Consequently, $f^{2}\left(t u_{1}+(1-t) u_{2}, y\right) \geq 0$, which implies that $t u_{1}+(1-t) u_{2} \in \Phi(x)$. Hence, $\Phi(x)$ is convex. We next claim that $\Phi(x)$ is closed. Let $\left\{u_{n}\right\} \subset \Phi(x)$ be a sequence converging to $w$. Then, $f^{2}\left(u_{n}, y\right) \geq 0$ for all $y \in Z(x)$. From the upper semicontinuous of $f^{2}(\cdot, y)$ at $u$, we have

$$
f^{2}(u, y) \geq 0 \text {. }
$$

Thus, $u \in \Phi(x)$, i.e., $\Phi(x)$ is closed. Finally, we prove that $\Phi$ is upper semicontinuous on $S_{\mathrm{EP}_{1}}$. Suppose to the contrary that $\Phi$ is not usc at some point $x_{0} \in S_{\mathrm{EP}_{1}}$. Then, there exists a neighborhood $U$ of $\Phi\left(x_{0}\right)$ such that there are $x_{n}$ converging to $x_{0}$ and $u_{n} \in \Phi\left(x_{n}\right)$ with $u_{n} \notin U$ for all $n$. Since $S_{\mathrm{EP}_{1}}$ is compact, $u_{n}$ (take a subsequence if necessary) converge to some point $u_{0} \in S_{\mathrm{EP}_{1}}$. For each $y_{0} \in Z\left(x_{0}\right)$, the lower semicontinuity of $Z$ ensures the existence of $y_{n} \in Z\left(x_{n}\right)$ with $y_{n} \rightarrow y_{0}$. Since $u_{n} \in \Phi\left(x_{n}\right)$, we have $f^{2}\left(u_{n}, y_{n}\right) \geq 0$. It follows from the upper semicontinuous of $f^{2}$ at $\left(u_{0}, y_{0}\right)$ that $f^{2}\left(u_{0}, y_{0}\right) \geq 0$, i.e., $w_{0} \in \Phi\left(x_{0}\right)$, which is a contradiction as $u_{n} \notin U$ for all $n$. Hence, $\Phi$ is usc on $S_{\mathrm{EP}_{1}}$. By Kakutani-Fan-Glicksberg fixed point theorem, there exists $x \in S_{\mathrm{EP}_{1}}$ such that $\Phi(x)=x$. Therefore, $S_{\mathrm{LEP}}$ is nonempty. The compactness of the set of solutions is obvious.

Remark 3.1. In [9], Bianchi, Konnov and Pini proposed an approach the existence of solutions of (LEP) via the analysis of the solutions of sequential equilibrium problems. Theorem 3.1 is a direct investigation for the existence of solutions of (LEP). In this way, we have to utilize the lower semicontinuity of the 
set-valued mapping $Z$. The following result provides sufficient conditions for the mentioned property of $Z$.

Proposition 3.5. Assume that $S_{\mathrm{EP}_{1}}$ is a nonempty compact set and

(i) $f^{1}(\cdot, y)$ is strictly quasiconcave and lower semicontinuous for each $y \in K$;

(ii) $f^{1}$ is pseudomonotone and is a transfer weak pseudo-distance on $K \times K$.

Then, $Z$ is lsc on $S_{\mathrm{EP}_{1}}$.

Proof. For each $\bar{x} \in S_{\mathrm{EP}_{1}}$, let $\bar{z}$ be an arbitrary point in $Z(\bar{x})$. It is clear that $f^{1}(\bar{x}, \bar{z})=0$ and $f^{1}(\bar{x}, y) \geq 0$ for all $y \in K$. Because $f^{1}$ is a transfer weak pseudo-distance on $K \times K$, we have $f^{1}(\bar{z}, y) \geq 0$, which implies that $\bar{z} \in S_{\mathrm{EP}_{1}}$. It follows from the nonemptiness and compactness of $S_{\mathrm{EP}_{1}}$ that there exists a sequence of points $z_{n} \in S_{\mathrm{EP}_{1}}$ converging to $\bar{z}$. We claim that $z_{n} \in Z\left(x_{n}\right)$ for every $x_{n} \in S_{\mathrm{EP}_{1}}$. Indeed, since $z_{n} \in S_{\mathrm{EP}_{1}}$, $f^{1}\left(z_{n}, x_{n}\right) \geq 0$. Combining this and the pseudomonotonicity of $f^{1}$ gives us $f^{1}\left(x_{n}, z_{n}\right) \leq 0$, which yields that $f_{1}\left(x_{n}, z_{n}\right)=0$, as $x_{n} \in S_{\mathrm{EP}_{1}}$. Therefore, $z_{n} \in Z\left(x_{n}\right)$, this brings the proof to its end.

The following result is an alternative approach for the existence solutions of (LEP).

\section{Theorem 3.2. Assume that}

(i) $f^{1}(\cdot, y)$ is quasiconcave for each $y \in K$;

(ii) $f^{1}$ is a transfer weak pseudo-distance on $K \times K$;

(iii) $f^{1}$ and $f^{2}$ are properly quasimonotone on $K \times K$;

(iv) $f^{1}(\cdot, y)$ and $f^{2}(\cdot, y)$ are upper semicontinuous for each $y \in K$.

Then, the set $S_{\mathrm{LEP}}$ is nonempty.

Proof. It follows immediately from Proposition 3.4 that $S_{\mathrm{EP}_{1}}$ is nonempty, compact and convex. By setting

$$
T:=\left\{x \in S_{\mathrm{EP}_{1}} \mid f^{2}(x, y) \geq 0, \forall y \in S_{\mathrm{EP}_{1}}\right\}
$$

and applying Proposition 3.4 again we obtain that $T$ is nonempty. For every $x \in S_{\mathrm{EP}_{1}}$, let $z$ be an arbitrary point in $Z(x)$. Because $f^{1}$ is a transfer weak pseudo-distance and $f^{1}(x, z)=0$, it yields from the fact $f^{1}(x, y) \geq 0$ for all $y \in K$ that $f^{1}(z, y) \geq 0$. Thus, $z \in S_{\mathrm{EP}_{1}}$, i.e., $Z(x) \subset S_{\mathrm{EP}_{1}}$. Consequently, $T \subset S_{\mathrm{LEP}}$. The proof is complete.

Remark 3.2. Theorem 3.2 is strictly improve Theorem 2 in [9]. Namely, the weak pseudo-distance assumption on $f^{1}$ is relaxed by the transfer weak pseudo-distance.

\section{Convergence CONDitions}

In this section, we mainly study the Painlevé-Kuratowski convergence of the solution sets of a family of perturbed lexicographic vector equilibrium problems by perturbing both the constraint set and the objective function. We investigate this convergence by using the Painlevé-Kuratowski convergence of the sequence of the perturbed constraint sets and the continuous convergence of the sequence of the perturbed objective functions. In this regard, we firstly establish the following result.

Lemma 4.1. Assume that

(i) $K_{n} \stackrel{\text { P.K. }}{\longrightarrow} K$ and $f_{n}^{1} \stackrel{\mathrm{c}}{\rightarrow} f^{1}$; 
(ii) The Fréchet derivative, $\nabla_{2} f^{1}$, of $f^{1}$ with respect to the second argument exists and $\nabla_{2} f^{1}(x, \cdot)$ is surjective on $X \backslash\{x\}$ for each $x \in X$.

Then, for each $x_{n} \rightarrow x$ and $z \in Z(x) \backslash\{x\}$, there exist $z_{n} \in Z_{n}\left(x_{n}\right)$ such that $z_{n} \rightarrow z$.

Proof. For each $x_{n} \rightarrow \bar{x}$ and $\bar{z} \in Z(\bar{x}) \backslash\{\bar{x}\}$, we claim that for each neighborhood $V$ of $\bar{z}, V \subset X$, there exist a neighborhood $U$ of $\bar{x}$ and a function $s_{n}: U \rightarrow V$ such that $s_{n}(x) \in Z_{n}(x)$ for all $x \in U$. Let $m=$ $\left\|\nabla_{2} f^{1}(\bar{x}, \bar{z})^{-1}\right\|$ and $\alpha$ be a positive real number such that $B_{\alpha}(\bar{z}) \subset V$. From the Fréchet differentiability of $f^{1}$, one can choose a real number $\beta \in(0, \alpha]$ such that

$$
\left|f^{1}(\bar{x}, z)-f^{1}(\bar{x}, \bar{z})-\left\langle\nabla_{2} f^{1}(\bar{x}, \bar{z}), z-\bar{z}\right\rangle\right| \leq \frac{1}{2 m}\|z-\bar{z}\|, \forall z \in B_{\beta}(\bar{z}),
$$

and consequently,

$$
\left|f^{1}(\bar{x}, z)-\left\langle\nabla_{2} f^{1}(\bar{x}, \bar{z}), z-\bar{z}\right\rangle\right| \leq \frac{\beta}{2 m}, \quad \forall z \in B_{\beta}(\bar{z}) .
$$

Since $f_{n}^{1} \stackrel{\mathrm{c}}{\rightarrow} f^{1}$, there exists a positive real number $\gamma \leq \beta$ such that for all $x \in B_{\gamma}(\bar{x}), z \in B_{\beta}(\bar{z})$, one has

$$
\left|f_{n}^{1}(x, z)-f^{1}(\bar{x}, z)\right| \leq \frac{\beta}{2 m}
$$

For each $x \in B_{\gamma}(\bar{x})$, we construct the function $\xi_{x}^{(n)}: B_{\beta}(\bar{z}) \rightarrow X$ satisfying

$$
\xi_{x}^{(n)}(z)=\nabla_{2} f^{1}(\bar{x}, \bar{z})^{-1}\left(\left\langle\nabla_{2} f^{1}(\bar{x}, \bar{z}), z\right\rangle-f_{n}^{1}(x, z)\right) .
$$

Obviously, $\xi_{x}^{(n)}$ is continuous in $B_{\beta}(\bar{z})$. Further, for any $z \in B_{\beta}(\bar{z})$, one has

$$
\begin{aligned}
\left\|\xi_{x}^{(n)}(z)-\bar{z}\right\| & =\left\|\nabla_{2} f^{1}(\bar{x}, \bar{z})^{-1}\left(\left\langle\nabla_{2} f^{1}(\bar{x}, \bar{z}), z\right\rangle-f_{n}^{1}(x, z)\right)-\bar{z}\right\| \\
& =\left\|\nabla_{2} f^{1}(\bar{x}, \bar{z})^{-1}\right\|\left|\left\langle\nabla_{2} f^{1}(\bar{x}, \bar{z}), z-\bar{z}\right\rangle-f_{n}^{1}(x, z)\right| \\
& \leq m\left(\left|\left\langle\nabla_{2} f^{1}(\bar{x}, \bar{z}), z-\bar{z}\right\rangle-f^{1}(\bar{x}, z)\right|+\left|f^{1}(\bar{x}, z)-f_{n}^{1}(x, z)\right|\right) \\
& \leq m\left(\frac{\beta}{2 m}+\frac{\beta}{2 m}\right)=\beta .
\end{aligned}
$$

Hence, thanks to the Schauder's fixed-point theorem, for all $x \in B_{\gamma}(\bar{x})$, there exists a point $s_{n}(x) \in B_{\beta}(\bar{z}) \subset$ $V$ such that $\xi_{(x)}^{(n)}\left(s_{n}(x)\right)=s_{n}(x)$. Thus,

$$
s_{n}(x)=\nabla_{2} f^{1}(\bar{x}, \bar{z})^{-1}\left(\left\langle\nabla_{2} f^{1}(\bar{x}, \bar{z}), s_{n}(x)\right\rangle-f_{n}^{1}\left(x, s_{n}(x)\right)\right),
$$

which is equivalent to $f_{n}^{1}\left(x, s_{n}(x)\right)=0$, i.e. $s_{n}(x) \in Z_{n}(x)$.

We next focus on the upper part of the convergence of the solution sets $S_{\mathrm{LEP}}^{n}$.

Theorem 4.1. Assume that

(i) $K_{n} \stackrel{\text { P.K. }}{\longrightarrow} K$ and $f_{n}^{i} \stackrel{\text { c }}{\rightarrow} f^{i}$, for $i=1,2$;

(ii) $f^{1}(x, \cdot)$ and $f^{2}(x, \cdot)$ are explicitly quasiconvex for each $x \in K$;

(iii) $f^{1}$ and $f^{2}$ are pseudomonotone;

(iv) The Fréchet derivative, $\nabla_{2} f^{1}$, of $f^{1}$ with respect to the second argument exists and $\nabla_{2} f^{1}(x, \cdot)$ is surjective on $X \backslash\{x\}$.

Then, the solution set of (LEP) is nonempty and $\lim _{\sup }{ }_{n \rightarrow \infty} S_{\mathrm{LEP}}^{n} \subset S_{\mathrm{LEP}}$. 
Proof. The nonemptiness of the solution set $S_{\mathrm{LEP}}$ follows from Theorem 3.1. We show that $\lim _{\sup _{n \rightarrow \infty}} S_{\mathrm{LEP}}^{n}$ $\subset S_{\text {LEP. Let }} x_{0}$ be an arbitrary point of $\limsup _{n \rightarrow \infty} S_{\text {LEP }}^{n}$. Then, there exists a subsequence $\left\{x_{n_{k}}\right\}$ in $S_{\text {LEP }}^{n_{k}}$ such that $x_{n_{k}} \rightarrow x_{0}$. Since $K_{n_{k}} \stackrel{\text { P.K. }}{\longrightarrow} K$, we have $x_{0} \in K$. Because $x_{n_{k}} \in S_{\text {LEP }}^{n_{k}}$,

$$
\begin{cases}f_{n_{k}}^{1}\left(x_{n_{k}}, y\right) \geq 0, & \forall y \in K_{n_{k}}, \\ f_{n_{k}}^{2}\left(x_{n_{k}}, z\right) \geq 0, & \forall z \in Z_{n_{k}}\left(x_{n_{k}}\right) .\end{cases}
$$

For each $y \in K$, as $K_{n_{k}} \stackrel{\text { P.K. }}{\longrightarrow} K$, there are $y_{n_{k}} \in K_{n_{k}}$ such that $y_{n_{k}} \rightarrow y$. It follows from (4.1) and the continuous convergence of $f^{1}$ that $f^{1}\left(x_{0}, y\right) \geq 0$. If $x_{0} \notin S_{\mathrm{LEP}}$, then there is $z_{0} \in Z\left(x_{0}\right)$ such that

$$
f^{2}\left(x_{0}, z_{0}\right)<0 .
$$

Applying Lemma 3.5, there exist $z_{n_{k}} \in Z_{n_{k}}\left(x_{n_{k}}\right)$ such that $z_{n_{k}} \rightarrow z_{0}$. Combining this with (4.2) and taking into account the continuous convergence of $f^{2}$, we arrive at $f^{2}\left(x_{0}, z_{0}\right) \geq 0$, which contradicts (4.3). Therefore, $x_{0} \in S_{\mathrm{LEP}}$, this brings the proof to the end.

The essentialness of all assumptions is now explained by the following counterexamples.

Example 4.1. (The Painlevé-Kuratowski convergence of constraint sets is essential) Let $X=\mathbb{R}, K=$ $[-1,1], K_{n}=\left[-2-n^{-1}, 2+n^{-1}\right]$. Let $f, f_{n}: X \times X \rightarrow \mathbb{R}^{2}$ be the functions defined, respectively, by

$$
f(x, y)=(y-x, y-x) \text { and } f_{n}(x, y)=\left(\left(1+\frac{1}{n}\right)(y-x),\left(1+\frac{1}{n}\right)(y-x)\right) .
$$

Obviously, assumption (ii) and the lower part of convergence of $K_{n}$ are satisfied while the upper part of convergence is not. We have $S_{\mathrm{LEP}}=\{-1\}$ and $S_{\mathrm{LEP}}^{n}=\left\{-2-n^{-1}\right\}$; however, $\{-2\}=\limsup _{n \rightarrow \infty} S_{\mathrm{LEP}}^{n} \not \subset$ $S_{\text {LEP }}=\{-1\}$.

Example 4.2. (The continuous convergence of objective function is essential) Let $X=\mathbb{R}^{2}, K=[0,2]^{2}$ and $f(x, y)=\left(f^{1}(x, y), f^{2}(x, y)\right)$ defined by

$$
f^{1}(x, y)=y_{1}-x_{1}, \quad f^{2}(x, y)= \begin{cases}x_{2}-y_{2}, & \text { if } x_{2} \leq 1 \\ 0, & \text { if } x_{2}>1\end{cases}
$$

It is clear that assumption (ii) is satisfied. Direct computations give us $S_{\mathrm{LEP}}=\left\{\left(0, x_{2}\right) \mid 1<x_{2} \leq 2\right\}$. Let $K_{n}=[0,2]^{2}$, and $f_{n}(x, y)=\left(f_{n}^{1}(x, y), f_{n}^{2}(x, y)\right)$, where $f_{n}^{1}(x, y)=n^{-1}\left(x_{2}-y_{2}\right)^{2}+y_{1}-x_{1}$, and

$$
f_{n}^{2}(x, y)= \begin{cases}\left(x_{2}-y_{2}\right) \exp \left(\frac{x_{1}-y_{1}}{n}\right), & \text { if } x_{2} \leq 1, \\ \left(x_{1}-y_{1}\right)\left(\exp \left(\frac{x_{2}-y_{2}}{n}\right)-1\right), & \text { if } x_{2}>1 .\end{cases}
$$

Let $x_{n}=\left(0,(n+1) n^{-1}\right)$. It is not hard to verify that $\left\{x_{n}\right\} \subset S_{\mathrm{LEP}}^{n}$. But $x_{n} \rightarrow(0,1) \notin S_{\mathrm{LEP}}$. Hence, $\limsup _{n \rightarrow \infty} S_{\text {LEP }}^{n} \not \subset S_{\text {LEP. }}$. The reason is that $f_{n}^{2}$ does not converge continuously to $f^{2}$. Indeed, taking $x_{n}=\left(1,(n+1) n^{-1}\right), y_{n}=(1,2)$, we have $x_{n} \rightarrow x=(1,1), y_{n} \rightarrow y=(1,2)$ and $f_{n}^{2}\left(x_{n}, y_{n}\right)=0$, but $f_{2}(x, y)=$ $-1<0$.

Example 4.3. (The surjectivity of $\nabla_{2} f^{1}$ cannot be dispensed) Let $X=\mathbb{R}, K=[0,1], K_{n}=\left[-n^{-1}, 1+\right.$ $n^{-1}$. Consider the functions $f, f_{n}: X \times X \rightarrow \mathbb{R}^{2}$ defined as $f(x, y)=\left(x(x-y)^{2}, \exp (y)(x-y)\right)$, and

$$
f_{n}(x, y)=\left(\left(x+\frac{1}{n}\right)(x-y)^{2},\left(1+\frac{y}{n}\right)^{n}(x-y)\right) .
$$


Obviously, assumption (i) is satisfied. Direct computations give us $S_{\mathrm{LEP}}=(0,1]$ and $S_{\mathrm{LEP}}^{n}=\left(-n^{-1}, 1+\right.$ $\left.n^{-1}\right]$. Hence, $\limsup _{n \rightarrow \infty} S_{\mathrm{LEP}}^{n} \not \subset S_{\mathrm{LEP}}$. This is due to the violation of assumption (ii) as $\nabla_{2} f^{1}(0, y)=0$, for all $y \in X$.

Now we can state the main result about the convergence in the sense of Painlevé-Kuratowski for the solutions of the perturbed problems.

Theorem 4.2. Impose all assumptions of Theorem 4.1 and assume further that

(iii) $K$ is convex;

(iv) $f^{1}(\cdot, y)$ is strictly quasiconcave on $K$, for all $y \in K$.

Then, $S_{\mathrm{LEP}}^{n}$ converge to $S_{\mathrm{LEP}}$ in the sense of Painlevé-Kuratowski.

Proof. We need only to prove that $S_{\mathrm{LEP}} \subset \liminf S_{\mathrm{LEP}}^{n}$. Suppose to the contrary that, there exists $x_{0} \in S_{\mathrm{LEP}}$ such that $x_{0} \notin \liminf S_{\mathrm{LEP}}^{n}$. Then, there is a neighborhood $U_{0}$ of the origin in $X$ such that

$$
S_{\mathrm{LEP}}^{n} \cap\left(x_{0}+U_{0}\right)=\emptyset \text { for } n \text { sufficiently large } .
$$

We discuss the following two cases:

Case 1. $S_{\mathrm{LEP}}=\left\{x_{0}\right\}$. Let $\left\{x_{n}\right\}$ be an arbitrary sequence in $S_{\mathrm{LEP}}^{n}$. Since $K_{n} \stackrel{\text { P.K. }}{\longrightarrow} K$, one can assume that $x_{n} \rightarrow \bar{x}_{0}$ for some $\bar{x}_{0} \in K$. The same arguments given in the proof of Theorem 4.1 show that $\bar{x}_{0} \in S_{\mathrm{LEP}}$. Noting the fact that $S_{\mathrm{LEP}}$ is a singleton, we have $x_{0}=\bar{x}_{0}$, and so $x_{n} \rightarrow \bar{x}_{0}=x_{0}$. Thus, $x_{n} \in x_{0}+U_{0}$ for $n$ large enough. This together with $x_{n} \in S_{\mathrm{LEP}}^{n}$ imply that $S_{\mathrm{LEP}}^{n} \cap\left(x_{0}+U_{0}\right) \neq \emptyset$, which contradicts (4.4).

Case 2. There exists $\bar{x} \in S_{\mathrm{LEP}}$ satisfying $\bar{x} \neq x_{0}$. Then, for any $y \in K$, one has $f^{1}\left(x_{0}, y\right) \geq 0$ and $f^{1}(\bar{x}, y) \geq$ 0 . By the strict quasiconcavity of $f^{1}(\cdot, y)$ on $K$, one has

$$
f^{1}\left(t \bar{x}+(1-t) x_{0}, y\right)>\min \left\{f^{1}(\bar{x}, y), f^{1}\left(x_{0}, y\right)\right\} \geq 0, \forall t \in(0,1) .
$$

Putting $x(t):=t \bar{x}+(1-t) x_{0}$, we have $x(t) \in K$. It is worth noting that for the chosen $U_{0}$, there exist a neighborhood $U_{1}$ of the origin in $X$ and $t_{0} \in(0,1)$ satisfying $U_{1}+U_{1} \subset U_{0}$ and $x\left(t_{0}\right) \in x_{0}+U_{1}$. Hence, $x\left(t_{0}\right)+U_{1} \subset x_{0}+U_{0}$. Since $x\left(t_{0}\right) \in K$ and $K_{n} \stackrel{\text { P.K. }}{\longrightarrow} K$, there are $\bar{x}_{n}\left(t_{0}\right) \in K_{n}$ with $\bar{x}_{n}\left(t_{0}\right) \rightarrow x\left(t_{0}\right)$. Thus, $\bar{x}_{n}\left(t_{0}\right) \in x\left(t_{0}\right)+U_{1} \subset x_{0}+U_{0}$, for $n$ sufficiently large. Combining this with (4.4), one has $\bar{x}_{n}\left(t_{0}\right) \notin S_{\mathrm{LEP}}^{n}$, for $n$ large enough. Hence, there exist $\bar{y}_{n} \in K_{n}$ such that

$$
f_{n}^{1}\left(\bar{x}_{n}\left(t_{0}\right), \bar{y}_{n}\right)<0 \text {. }
$$

According to Lemma 2.1, we can assume that $\left\{\bar{y}_{n}\right\}$ (taking a subsequence if necessary) converges to some $\bar{y} \in K$. It follows from (4.6) and the continuous convergence of $f_{n}^{1}$ that $f^{1}\left(x\left(t_{0}\right), \bar{y}\right) \leq 0$, which contradicts (4.5). This brings the proof to the end.

We give an example to illustrate that the strict quasiconcavity assumption on $f^{1}$ is essential.

Example 4.4. Let $X=\mathbb{R}, K=[0,1]$ and $K_{n}=\left[\exp \left(n^{-1}\right)-1, \exp \left(2 n^{-1}\right)\right]$. Consider the functions $f, f_{n}$ : $X \times X \rightarrow \mathbb{R}^{2}$ defined as

$$
f(x, y)=\left((x-y)^{2},(x-y) \exp (x y)\right),
$$

and

$$
f_{n}(x, y)=\left((x-y)\left(x-y-\frac{1}{n}\right),\left(1+\frac{x y}{n}\right)^{n}(x-y)\right) .
$$

It is obvious that assumptions (i)-(iii), except (iv), are satisfied. Direct computations give us $S_{\mathrm{LEP}}=[0,1]$ and $S_{\mathrm{LEP}}^{n}=\left\{\exp \left(n^{-1}\right)-1\right\}$. Hence, $S_{\mathrm{LEP}}^{n}$ does not converge to $S_{\mathrm{LEP}}$ in the sense of Painlevé-Kuratowski. 


\section{APPLICATIONS TO LEXICOGRAPHIC VARIATIONAL INEQUALITIES}

Let $X, X^{*}$ and $K$ be as in the preceding sections, and $A^{i}: X \rightarrow X^{*}$, for $i=1,2$. We consider the following lexicographic variational inequality.

(LVI) Find $\bar{x} \in K$ such that

$$
\left(\left\langle A^{1}(\bar{x}), y-\bar{x}\right\rangle,\left\langle A^{2}(\bar{x}), y-\bar{x}\right\rangle\right) \geq_{L} 0, \quad \forall y \in K .
$$

The family of perturbed lexicographic variational inequalities (LVI) ${ }_{n}, n \in \mathbb{N}$ is given as $(\mathrm{LVI})_{n}$ Find $\bar{x}_{n} \in K_{n}$ such that

$$
\left(\left\langle A_{n}^{1}\left(\bar{x}_{n}\right), y-\bar{x}\right\rangle,\left\langle A_{n}^{2}\left(\bar{x}_{n}\right), y-\bar{x}\right\rangle\right) \geq_{L} 0, \quad \forall y \in K_{n},
$$

where $K_{n} \subset X$ and $A_{n}^{i}: X \rightarrow X^{*}$.

The following results are derived directly from the corresponding ones in Section 4 by setting $f^{i}(x, y)=$ $\left\langle A^{i}(x), y-x\right\rangle$ for $i=1,2$.

Corollary 5.1. For (LVI), assume that the following conditions hold:

(i) $K_{n} \stackrel{\text { P.K. }}{\rightarrow} K$ and $A_{n}^{i} \stackrel{\mathrm{c}}{\rightarrow} A^{i}$ for $i=1,2$;

(ii) $A^{1}$ is surjective.

Then, $\lim \sup _{n \rightarrow \infty} S_{\mathrm{LVI}}^{n} \subset S_{\mathrm{LVI}}$.

Corollary 5.2. For (LVI), impose the assumptions of Corollary 5.1 and the additional conditions:

(iii) $K$ is convex;

(iv) the function $x \mapsto\left\langle A^{1}(x), y-x\right\rangle$ is strictly quasiconcave on $K$, for all $y \in K$.

Then, $S_{\mathrm{LVI}}^{n}$ converge to $S_{\mathrm{LVI}}$ in the sense of Painlevé-Kuratowski.

\section{Acknowledgments}

This research was funded by Vietnam National Foundation for Science and Technology Development (NAFOSTED) under Grant Number 101.01-2017.18.

\section{REFERENCES}

[1] B. Alleche, V.D. Rădulescu, M. Sebaoui, The Tikhonov regularization for equilibrium problems and applications to quasihemivariational inequalities, Optim. Lett. 9 (2015), 483-503.

[2] L.Q. Anh, T.Q. Duy, A.Y. Kruger, N.H. Thao, Well-posedness for lexicographic vector equilibrium problems, in: V.F. Demyanov et al. (eds.), Constructive Nonsmooth Analysis and Related Topics, vol. 87, pp. 159-174, Springer, New York, 2014.

[3] L.Q. Anh, T.Q. Duy, P.Q. Khanh, Continuity properties of solution maps of parametric lexicographic equilibrium problems, Positivity 20 (2016), 61-80.

[4] L.Q. Anh, T.Q. Duy, Tykhonov well-posedness for lexicographic equilibrium problems, Optimization 65 (2016), 19291948.

[5] L.Q. Anh, T.Q. Duy, On penalty method for equilibrium problems in lexicographic order, Positivity 22 (2018), $39-57$.

[6] M. Bianchi, R. Pini, A note on stability for parametric equilibrium problems, Oper. Res. Lett. 31 (2003), 445-450.

[7] M. Bianchi, R. Pini, Coercivity conditions for equilibrium problems, J. Optim. Theory Appl. 124 (2005), 79-92.

[8] M. Bianchi, I.V. Konnov, R. Pini, Lexicographic variational inequalities with applications, Optimization 56 (2007), 355367. 
[9] M. Bianchi, I.V. Konnov, R. Pini, Lexicographic and sequential equilibrium problems, J. Glob. Optim. 146 (2010), 551560 .

[10] E. Blum, W. Oettli, From optimization and variational inequalities to equilibrium problems, Math. Student India 63 (1994), 123-145.

[11] P. Chatterjee, C.S. Lalitha, Scalarization of Levitin-Polyak well-posedness in vector optimization using weak efficiency, Optim. Lett. 9 (2015), 329-343.

[12] G. P. Crespi, M. Papalia, M. Rocca, Extended well-posedness of vector optimization problems: the convex case, Taiwanese J. Math. 15 (2011), 1545-1559.

[13] M. Durea, On the existence and stability of approximate solutions of perturbed vector equilibrium problems, J. Math. Anal. Appl. 333 (2007), 1165-1179.

[14] K. Fan, A Minimax Inequality and Applications, in: O. Shisha, ed., Inequalities III, pp. 103-113, Academic Press, New York, 1972.

[15] A. Göpfert, H. Riahi, C. Tammer, C. Zalinescu, Variational Methods in Partially Ordered Spaces, Springer, Berlin, 2003.

[16] I.L. Glicksberg, A further generalization of the Kakutani fixed point theorem, with application to nash equilibrium points, Proc. Amer. Math. Soc. 3 (1952), 170-174.

[17] N. Hadjisavvas, S. Schaible, On strong pseudomonotonicity and (semi)strict quasimonotonicity, J. Optim. Theory Appl. 79 (1993), 139-155.

[18] I.V. Konnov, On lexicographic vector equilibrium problems, J. Optim. Theory Appl. 118 (2003), 681-688.

[19] K. Kuratowski, Topology, Vol.1 and 2, Academic Press, New York, 1968.

[20] C.S. Lalitha, P. Chatterjee, Stability and scalarization in vector optimization using improvement sets, J. Optim. Theory Appl. 166 (2015), 825-843.

[21] X.B. Li, N.J. Huang, Generalized vector quasi-equilibrium problems on hadamard manifolds, Optim. Lett. 9 (2015), 155-170.

[22] X.B. Li, Z. Lin, Q.L. Wang, Stability of approximate solution mappings for generalized Ky Fan inequality, TOP 24 (2016), 196-205.

[23] M.B. Lignola, J. Morgan, V. Scalzo, Vector quasi-variational inequalities under perturbations, Pacific J. Optim. 2 (2006), 599-609.

[24] M.B. Lignola, J. Morgan, V. Scalzo, Lower convergence of approximate solutions to vector quasi- variational problems, Optimization 59 (2010), 821-832.

[25] E. Miglierina, E. Molho, Convergence of minimal sets in convex vector optimization, SIAM J. Optim. 15 (2005), $513-526$.

[26] H. Nikaidô, K. Isoda, Note on non-cooperative convex game, Pacific J. Math. 5 (1955), 807-815.

[27] Z.Y. Peng, X.B. Li, X.J. Long, X.D. Fan, Painleve-Kuratowski stability of approximate efficient solutions for perturbed semi-infinite vector optimization problems, Optim. Lett. 12 (2017), 1-18.

[28] E. Wolk, Continuous convergence in partially ordered sets, Gen. Topology Appl. 5 (1975), 221-234.

[29] J. Zeng, S.J. Li, W.Y. Zhang, X.W. Xue, Hadamard well-posedness for a set-valued optimization problem, Optim. Lett. 7 (2013), 559-573. 